\title{
Time Delay Compensation for a Class of Dual Loop Networked Control System with Different Sampling Rate
}

\author{
Hong Zhao, Ke Che and Shenglan Zhang \\ Mechanical and control engineering college, Guilin University of Technology, Guilin, China
}

\begin{abstract}
This paper investigates a class of dual loop networked control system with different sampling rate. Smith predictor is used to eliminate the influence of time delay. However, uncertain time-varying delay and different sampling rate in the actual production process make the system with conventional Smith predictor cannot achieve the desired control effect. A modified Smith predictor with regulator is proposed for this kind of networked control system, which can be used to overcome the problems caused by random network delay and various sampling rate. The simulation results based on MATLAB /Simulink show that the proposed model and method are efficient and feasible.
\end{abstract}

Keywords-different sampling rate; dual loop; networked control system; Smith predictor; time-varying delay

\section{INTRODUCTION}

With the rapid development of sensing, signal processing and communication technology, the research on networked control systems (NCSs) has attracted great attention. The application of NCSs is able to enhance the flexibility of control system, reduce human resource investment, and has convenient operation and maintenance. However, the existence of network delay will cause the performance of NCSs to decline, or even cause instability of the system ${ }^{[1]}$. If network delay is timevarying and random, then it will lead to system performance degradation, and the probability of instability increase ${ }^{[2]}$.

In order to improve the control quality of large delay systems, Smith ${ }^{[3]}$ proposed a model-based predictor compensation control method in 1957, which is also widely used in NCSs. Zhang ${ }^{[4]}$ proposed a design scheme of Smith predictor (SP) combined with neuron controller for single loop NCS. $\mathrm{Ma}^{[5]}$ surveyed the Smith predictive time delay compensation method by introducing fuzzy adaptive PID control for the randomness of NCS time delay. Wang ${ }^{[6]}$ deal with the design problem of combining SP with linear active disturbance rejection control for large time delay systems, and the stability conditions and parameter perturbation of the system are analyzed. So far most studies of NCSs focus on that of single loop or single sampling rate. However, the multi loop NCSs often have different sampling rate in the actual production process, such as dual loop control of robot speed and position, dual loop control of servo motor's displacement and armature current, dual loop control of single-axis speed and posture of aircraft ${ }^{[7]}$. With different the sampling rate of inner and outer loops, the performance of the system will also be affected, so it is necessary to compensate these negative effects by means of optimization.
Dual loop NCS with different sampling rate reflects the problem that the control system in production has a dual loop structure and the variable rate of controlled quantity is different ${ }^{[8]}$. In this paper, modified Smith predictor (MSP) is designed for a class of dual loop NCSs with different sampling rate, which can effectively overcome the effect of random network delay and has better control effect when the sampling rate of inner and outer loops are changed.

\section{SYSTEM STRUCTURE}

The structure of dual loop NCSs investigated in this paper is shown in Figure I. $R(s)$ and $C(s)$ are input and output of the system, $G_{c 1}(s)$ and $G_{c 2}(s)$ are the controller of outer and inner loop respectively, $G_{p 1}(s)$ is the main controlled object, $G_{p 2}(s)$ is the deputy controlled object, $T_{1}$ is the outer loop sampling period, $T_{2}$ is the inner loop sampling period. Network delay of the system is mainly composed of two parts: The transfer functions of the delay from the master controller $G_{c 1}(s)$ to the secondary loop and from the master controlled object $G_{p 1}(s)$ to the master controller $G_{c 1}(s)$ are $e^{-t_{c a} s}$ and $e^{-t_{s c} s}$ respectively.

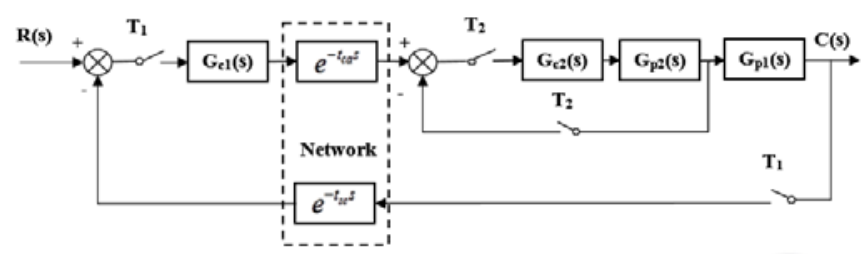

FIGURE I. STRUCTURE OF DUAL LOOP NCS WITH DIFFERENT SAMPLING RATE

The pulse transfer function of the system shown in Figure I can be written as

$$
\frac{C(\mathrm{z})}{R(\mathrm{z})}=\frac{\mathrm{z}^{-\frac{t_{c a}}{T_{1}}} G_{c 1}(\mathrm{z}) G_{c 2} G_{p 2} G_{p 1}(\mathrm{z})}{1+G_{c 2} G_{p 2}(\mathrm{z})+\mathrm{z}^{-\frac{t_{c a}}{T_{1}}} G_{c 1}(\mathrm{z}) G_{c 2} G_{p 1} G_{p 2}(z) z^{-\frac{t_{c c}}{T_{1}}}}
$$

It can be seen from Eq. (1) that there are delays in the denominator, which make the system is difficult to achieve the desired control effect. Therefore, the prediction model should be introduced to optimize system control. 


\section{DuAl LOOP NCS With DIFFERENT SAMPLING RATE BASED ON SP}

In this paper, the Smith prediction method is applied to dual loop NCS with different sampling rate. Now suppose the prediction model of the controlled object $G_{p 1}(s)$ and $G_{p 2}(s)$ are $G_{m 1}(s)$ and $G_{m 2}(s)$, the prediction model of network delay $e^{-t_{c a} s}$ and $e^{-t_{s c} s}$ are $e^{-t_{c a m} s}$ and $e^{-t_{s c m} s}$ respectively. According to dual loop control network structure shown in Figure I, the SP are added to the system, as shown in Figure II.

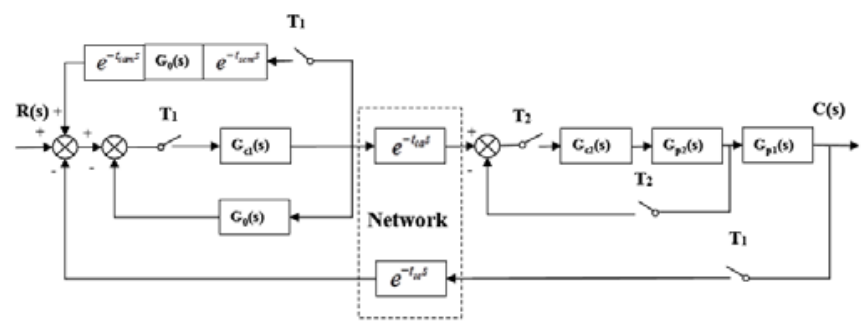

FIGURE II. STRUCTURE OF DUAL LOOP NCS WITH DIFFERENT SAMPLING RATE BASED ON SP

The transfer function of $G_{0}(s)$ can be written as

$$
G_{0}(s)=\frac{G_{m 1}(s) G_{m 2}(s) G_{c 2}(s)}{1+G_{m 2}(s) G_{c 2}(s)}
$$

The pulse transfer function of the system shown in Figure II is as follow

$$
\begin{aligned}
& \frac{C(z)}{R(z)}=\frac{z^{-\frac{t_{c a}}{T_{1}}} G_{c 1}(z) G_{c 2} G_{p 2} G_{p 1}(z)}{1+G_{A}+G_{B}-G_{C}} \\
& G_{A}=G_{c 1} G_{c 2} G_{m 1} G_{m 2}(z)+G_{c 2} G_{p 2}(z) \\
& G_{B}=z^{-\frac{t_{c a}}{T_{1}}} G_{c 1}(z) G_{c 2} G_{p 1} G_{p 2}(z) z^{-\frac{t_{c c}}{T_{1}}} \\
& G_{C}=z^{-\frac{t_{c a m}}{T_{1}}} G_{c 1}(z) G_{c 2} G_{m 1} G_{m 2}(z) z^{-\frac{t_{c c m}}{T_{1}}}
\end{aligned}
$$

When the prediction model of the controlled object $G_{m 1}(s)$ and $G_{m 2}(s)$ and that of the inner loop network delay $e^{-t_{c a m} s}$ and $e^{-t_{s m} s}$ achieve ideal condition: $G_{m 1}(s)=G_{p 1}(s), G_{m 2}(s)=G_{p 2}(s)$, $t_{c a m}=t_{c a}, t_{s c m}=t_{s c}$. That is, when the prediction model and the real model are equal, the denominator with the delay link is offset, then the pulse transfer function of system can be written as

$$
\frac{C(z)}{R(z)}=\frac{z^{-\frac{t_{c a}}{T_{1}}} G_{c 1}(z) G_{c 2} G_{p 2} G_{p 1}(z)}{1+G_{c 2} G_{p 2}(z)+G_{c 1}(z) G_{c 2} G_{p 1} G_{p 2}(z)}
$$

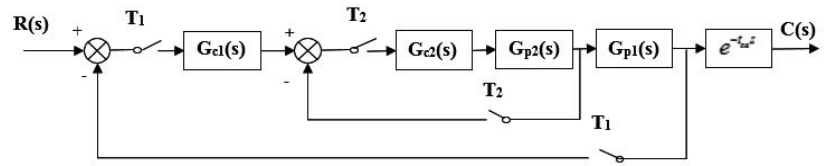

FIGURE III. STRUCTURE OF EQUIVALENT DUAL LOOP NCS BASED ON ACCURATE SP MODEL

Eq. (3) shows that the time delay of the closed-loop transfer function's denominator of the system is offset, and the structure of dual loop NCS can be equivalent to Figure III.

Figure III demonstrate that the effect of network delay on the stability of the system is reduced by dual loop NCS based on SP. The delay in the feedback loop of the system is offset, and network delay is removed from the system transfer function's denominator and becomes a pure time-delay link. While the pure time-delay link only causes the system response delay, it does not undermine the stability of the system.

\section{DuAl LoOp NCS With DifFERENT SAMPLING RATE BASED ON MSP}

In NCSs, there may be many different kinds of delays, which are not fixed, but random, time-varying and so on. It is difficult to predict accurately. In view of the above problems, this paper presents a design scheme of dual loop networks with different sampling rate compensated by MSP. The MSP adds a transfer function with an auxiliary regulator to the feedback channel, which requires lower model accuracy. Dual loop NCS based on MSP is shown in Figure IV.

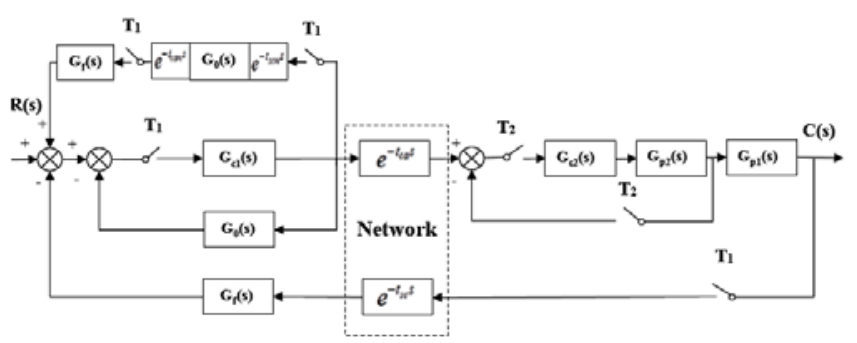

FIGURE IV. STRUCTURE OF EQUIVALENT DUAL LOOP NCS BASED ON MSP MODEL

The transfer function of $G_{0}(s)$ and $G_{f}(s)$ can be written as

$$
\begin{aligned}
& G_{0}(s)=\frac{G_{m 1}(s) G_{m 2}(s) G_{c 2}(s)}{1+G_{m 2}(s) G_{c 2}(s)} \\
& G_{\mathrm{f}}(s)=\frac{G_{c 2}(s) G_{0}(s)}{1+G_{c 2}(s) G_{0}(s)}
\end{aligned}
$$

The pulse transfer function of the system shown in Figure IV is as follow 


$$
\begin{aligned}
& \frac{C(z)}{R(z)}=\frac{G_{c 1}(z) G_{c 2} G_{p 2} G_{p 1}(z) z^{-\frac{t_{c a}}{T_{1}}}}{1+G_{A}+G_{B}-G_{C}} \\
& G_{A}=G_{c 1} G_{c 2} G_{m 1} G_{m 2}(z)+G_{c 2} G_{p 2}(z) \\
& G_{B}=z^{-\frac{t_{c a}}{T_{1}}} G_{c 1}(z) G_{c 2} G_{p 1} G_{p 2}(z) G_{f}(z) z^{-\frac{t_{s c}}{T_{1}}} \\
& G_{C}=z^{-\frac{t_{c a m}}{T_{1}}} G_{c 1}(z) G_{c 2} G_{m 1} G_{m 2}(z) G_{f}(z) z^{-\frac{t_{c c m}}{T_{1}}}
\end{aligned}
$$

When the prediction model of the controlled object $G_{m 1}(s)$ and $G_{m 2}(s)$ and that of the inner loop network delay $e^{-t_{c a m} s}$ and $e^{-t_{s c m} s}$ achieve ideal condition: $G_{m 1}(s)=G_{p 1}(s), G_{m 2}(s)=G_{p 2}(s)$, $t_{c a m}=t_{c a}, t_{c a}=t_{s c}$. When the prediction model and the real model are equal, the denominator with the delay link is offset, then the pulse transfer function of system can be written as Eq. (3).

\section{SYSTEM SimULATION}

The software MATLAB/Simulink is used as simulation tool. In order to verify the effect of network delay on the system, the system response analysis is carried out under different network delay in the NCS structure shown in Figure I. Network delay is assumed to be $t_{c a}=t_{s c}$, which are $0 \mathrm{~s}$, 3s and $10 \mathrm{~s}$ respectively. Considering NCS shown in Figure I, and assuming the transfer function of the controlled object $G_{p 1}(s)$ and $G_{p 2}(s)$ are $1 /(10 s+1)(s+1)^{2}$ and $1 /(30 s+1)$ respectively, $T_{1}$ is the sampling period of the outer loop and $T_{2}$ is the sampling period of the inner loop. Let $T_{1}=2 \mathrm{~s}$ and $T_{2}=1 \mathrm{~s}$. Then, Two parameters of control parameters are tuned respectively. The outer loop controller $G_{c 1}(s)$ adopts PI control and the proportion coefficient is $K_{\mathrm{p}}=1.38$, the integral coefficient is $T_{\mathrm{i}}=0.045$, the inner loop controller $G_{c 2}(s)$ adopts $\mathrm{P}$ control, and proportion coefficient is $K_{\mathrm{p}}=0.05$. The system input $\mathrm{R}$ is the unit step signal.

As shown from the Figure V that PID controller has better control effect without network delay. With the increase of network delay, dual loop NCS is significantly deteriorated.

In order to overcome the effect of network delay on the system, the conventional SP shown in Figure II is introduced to compensate the delay. Network delay $t_{c a}$ and $t_{s c}$ are all set to $10 \mathrm{~s}$, and the curves are shown in Figure VI. The unit step response curves of Y1 and Y0 of dual loop NCS based on SP and no SP are shown respectively.

As shown in Figure VI, the dynamic performance of Y1 is obviously better than that of Y0, which shows that SP can effectively compensate network delay and achieve better control effect when network delay can be accurately predicted.

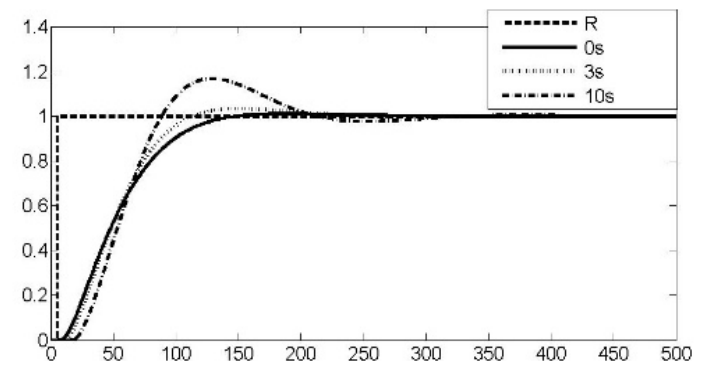

FIGURE V. NO SP (NETWORK DELAY IS 0S, 3S, 10S)

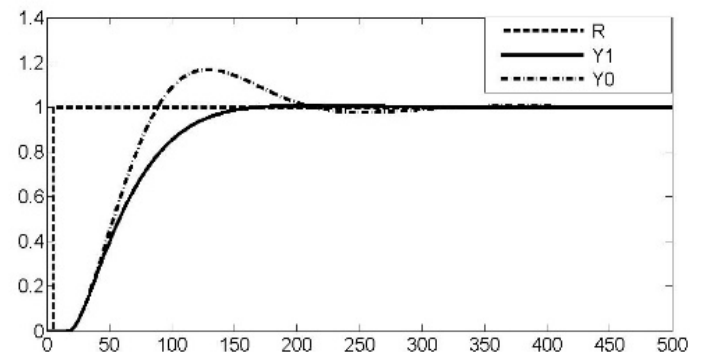

FIGURE VI. COMPARISON OF NO SP AND SP (NETWORK DELAY IS 10S)

In actual NCSs, network delay is random and difficult to accurately predict. Next, the unit step response curves of two cases with fixed delay and random delay are analyzed. The fixed delay is set to 10 s, and the Smith prediction delay model is set to $10 \mathrm{~s}$ at this time. Network delay is set as the fluctuation range from 1s to 19s, and the Smith prediction delay model is set to 10s. The unit step response curve can be obtained as shown in Figure VII. Y11 is the unit step response with fixed delay and Y12 is the unit step response with random delay.

As shown from the Figure VII, the control effect of dual loop NCS with different sampling rate based on SP is weakened when network delay is difficult to accurately predict. Therefore, MSP with regulator is introduced for the case that network delay difficult to accurately predict as shown from the Figure IV. Network delay is set as the fluctuation range from 1s to 19s, and the Smith prediction delay model is set to 10s. The unit step response curve is shown in Figure VIII. Y1 is the unit step response of dual loop NCS with different sampling rate of the SP and Y2 is the unit step response of dual loop NCS with different sampling rate of the MSP.

As shown from the Figure VIII that the effect of unit step response on $\mathrm{Y} 2$ is obviously less than $\mathrm{Y} 1$. In the case of random network delay and inaccurate network delay prediction, dual loop NCS based on MSP is not affected by the large range of delay variation, while the dynamic performance of dual loop NCS based on SP is significantly worse. It shows that the MSP achieves better compensation effect and better control effect than SP when network delay is difficult to accurately predict.

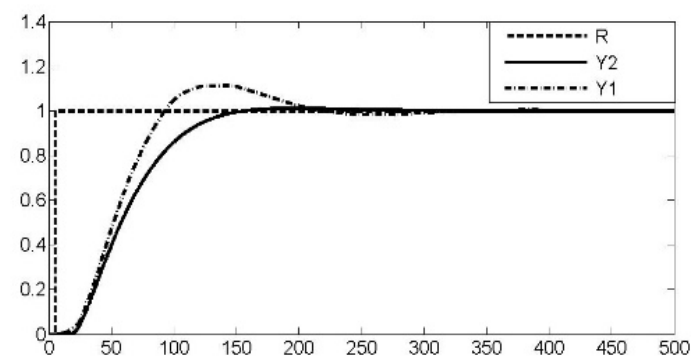

FIGURE VII. COMPARISON OF FIXED TIME DELAY AND RANDOM TIME DELAY 


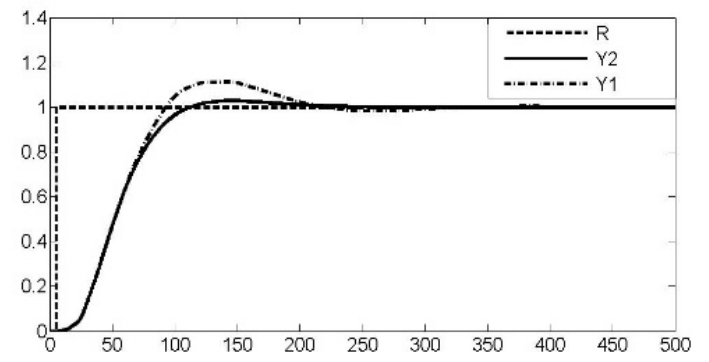

FIGURE VIII. COMPARISON OF MSP AND SP (RANDOM TIME DELAY)

According to the actual situation, the sampling ratio of inner and outer is often varied. Therefore, by changing the sampling ratio of inner and outer loops, the control effect of SP and MSP on dual loop NCS with different sampling rate is further compared. Network delay of SP model is 3s. The inner loop sampling time $T_{2}=1 \mathrm{~s}$, the outer loop sampling time $T_{1}$ are $2 \mathrm{~s}, 10 \mathrm{~s}$, 20s, 30s respectively. Network delay is set as the fluctuation range from $1 \mathrm{~s}$ to $5 \mathrm{~s}$. The unit step response of both is shown in Figure IX, X, XI and XII.

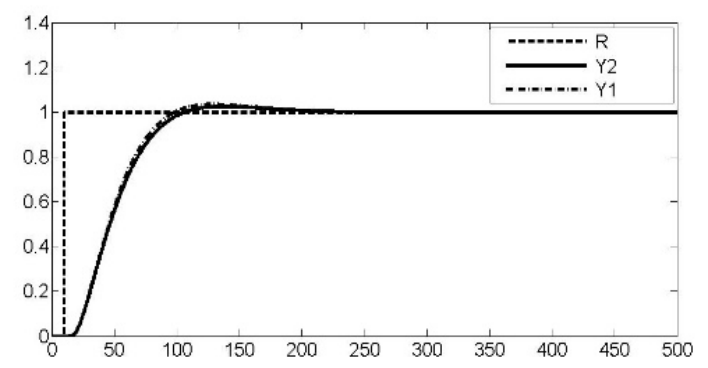

FIGURE IX. COMPARISON OF MSP AND SP $\left(T_{1}=2 \mathrm{~S}, T_{2}=1 \mathrm{~S}\right)$

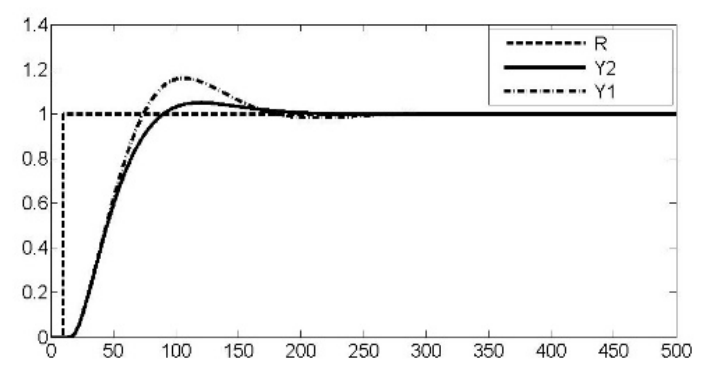

FIGURE X. COMPARISON OF MSP AND SP $\left(T_{1}=10 \mathrm{~S}, T_{2}=1 \mathrm{~S}\right)$

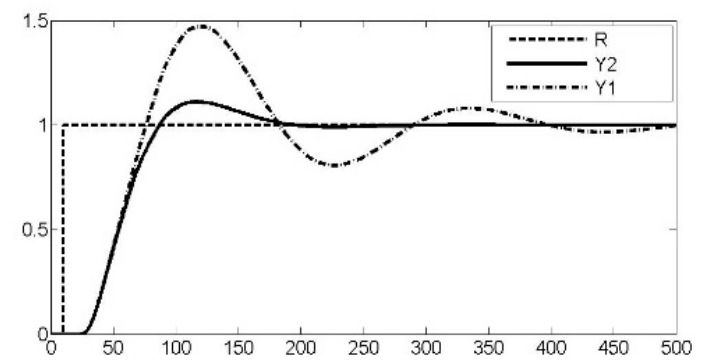

FIGURE XI. COMPARISON OF MSP AND SP $\left(T_{1}=20 \mathrm{~S}, T_{2}=1 \mathrm{~S}\right)$

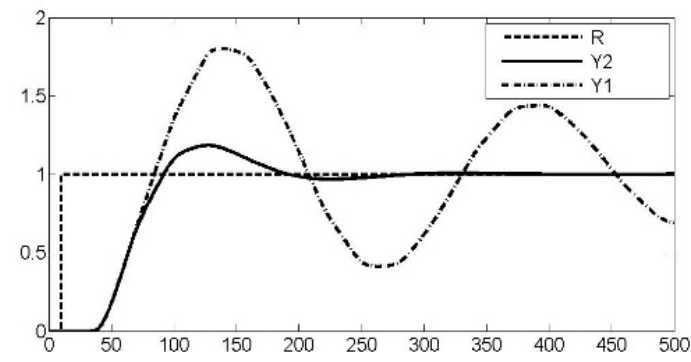

FIGURE XII. COMPARISON OF MSP AND SP $\left(T_{1}=30 S, T_{2}=1 \mathrm{~S}\right)$

Comparing with Figure IX, X, XI and XII, it can be concluded that $Y 2$ is still stable and unchanged with the increase of sampling rate of inner and outer loops, while Y1 becomes unstable gradually. Moreover, the rising time and adjusting time of Y2 are shorter and the overshoot is smaller under the same sampling rate of inner and outer loops, which indicates that the MSP is more stable. And the control effect of the system is better. When the sampling rate ratio of the inner and outer loop of dual loop NCS system reaches 30:1, dual loop NCS with different sampling rate based on SP is difficult to achieve balance. Dual loop NCS based on MSP is not affected by the change of the sampling rate between the inner and outer loops, but the dynamic performance of dual loop NCS based on SP is obviously worse. It is shown that dual loop NCS based on MSP can be applied to more systems with different sampling rate of inner and outer loops and has a wider application range than dual loop NCS based on SP.

\section{CONCLUSION}

In this paper, MSP is proposed for a class of dual loop NCSs with different sampling rate. The simulation results show that this method can overcome the effect of the random network delay when network delay is difficult to accurately predict, and has better control effect at different sampling rate of inner and outer loops than that of control system with conventional SP.

\section{ACKNOWLEDGMENT}

This work was financially supported by the Scientific Research Project of Guangxi Colleges and Universities (No. YB2014145), and Foundation of Guilin University of Technology.

\section{REFERENCES}

[1] Zhang W, Branieky M. S, \& Phillips S. M. (2016). Stability of Networked Control System. IEEE Control System Magazine, 21(l):84-99.

[2] You Keyou, Xie Lihua. The latest research on networked control systems. Journal of Automation, 2013, 39(2):111-118.

[3] Wang Jie, Zhang Xiaoqian. Time delay compensation for networked control systems. system simulation technology, 2017, 13(02):147-150.

[4] Li Guoyong, He Xiaogang, Yang Lijuan. Process control system. Beijing: Publishing House of electronics industry, 2017:190-191.

[5] Zhang Haitao, Li zhen. Simulation Study of Networked Control System Based on Smith Predictive Compensation. Computer Engineering and Applications, 2012, 08:243-245.

[6] Ma Yongguang, Jia Junru, Bo Junqing.Smith Predictive Time Delay Compensation for Networked Control Systems. Industrial Control Computer, 2014, 27(10):41-42. 
[7] Wang Yongshuai, Chen Qiang, Sun Mingwei, Sun Qinglin. Smith predictive auto-disturbance rejection control for first-order inertial largedelay systems. Acta Intelligent Systems, 2018, 08:1-10.

[8] Gu Cheng-cheng, Liu E-ting, Zhao Hong, Yang Lian. Modeling and Stability of a Class of Dual loop SISO Networked Control Systems with different sampling rate. Manufacturing Automation, 2013, 12:36-40.

[9] Liang Chenghe, Liu Electric Ting.Dual loop networked control system with packet loss and different sampling rate. Journal of Guilin University of Technology, 2015, 35(01):207-212. 